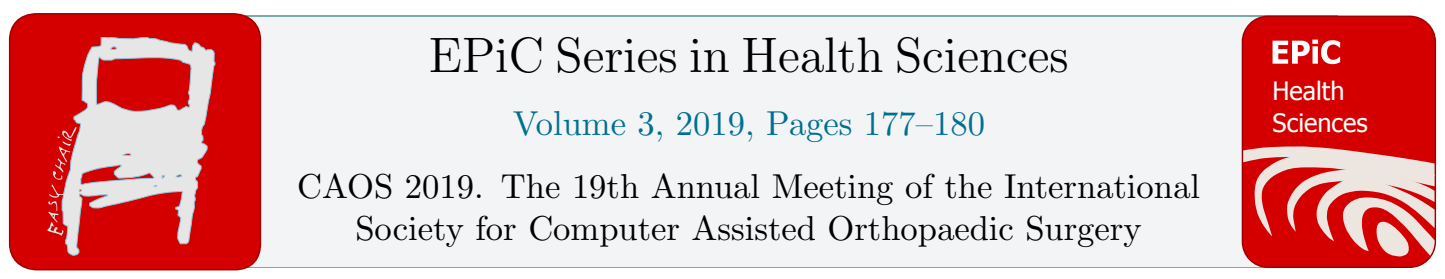

\title{
The interleaved partial active shape model (IPASM) search algorithm - towards 3D ultrasound-based bone surface reconstruction
}

\author{
Benjamin Hohlmann, Klaus Radermacher \\ Chair of Medical Engineering, Helmholtz Institute for Biomedical Engineering, RWTH Aachen \\ University, Germany \\ hohlmann@hia.rwth-aachen.de, radermacher@hia.rwth-aachen.de
}

\begin{abstract}
Several orthopedic applications require a three-dimensional model of the bone. Ultrasound is a radiation-free and cheap alternative to the state-of-the-art imaging modalities if its limitations in terms of image quality and viewing range can be overcome. This work presents in-vitro as well as in-vivo experiments evaluating the IPASM search, a method for combined segmentation, registration as well as extrapolation. The algorithm is capable to reconstruct the distal surface of a phantom femur with an average surface distance error of roughly $1 \mathrm{~mm}$ in case of in-vitro as well as below $2 \mathrm{~mm}$ for in-vivo records, even if the shape varies strongly from the initial model.
\end{abstract}

\section{Introduction}

In orthopedic surgery, several applications require a 3-dimensional model of the patient's bones: Pre-operative planning of prosthesis implantation, osteotomy or screw placement, biomechanical modelling and rapid prototyping for teaching and visualization purposes. In the Organisation for Economic Co-operation and Development (OECD) countries alone, there are 126 knee replacement surgeries per 100.000 inhabitants and year [OECD 2017], with an increasing number of operations utilizing jigs. The state of the art solutions for acquiring $3 \mathrm{D}$ surface models are computed tomography (CT) or magnetic resonance imaging (MRI). However, these imaging modalities are expensive and in the case of CT expose the patient to radiation. 3D ultrasound in contrast is a cheap and radiation-free imaging modality. Yet it suffers from a low signal-to-noise ratio, a limited field of view, the inability to see through highly reflective structures as well as several other image artifacts. These limitations aggravate the task of extracting a 3D-model of the bone from a simple segmentation in case of CT or MRI to a combined segmentation, registration as well as extrapolation in case of ultrasound.

All of these problems are thoroughly studied individually: In ultrasound segmentation bone enhancement methods achieved sub-millimeter accuracy [Hacihaliloglu 2017]. More recently, deep learning based approaches, building on before mentioned handcrafted features [Wang et al. 2018] as 
well as models built from scratch [Villa et al. 2018; Golan et al. 2016; Litjens et al. 2017] show promising results. Ultrasound registration algorithms often fuse US images with other imaging modalities, for example with a pre-operative CT in case of brain surgery [Masoumi et al. 2017]. Finally, extrapolation can be accomplished using statistical shape models [Anas et al. 2016]. Solving all of these problems simultaneously however remains a challenging task.

\section{Methods}

The IPASM search utilizes a statistical shape model (SSM) built from a database of 414 femora bones of varying gender and age. The algorithm alternates between rigid registration and shape adaption steps. For a more detailed description, please refer to [Hänisch et al. 2017]. We further enhanced the algorithm to compute two rigid registrations: One for the whole model, correcting bad initializations and a set of registrations for each individual image, enabling the algorithm to compensate tracking or patient movement errors.

The in-vitro test setup consist of a 3D US imaging system by Ultrasonix with a mechanically swiping probe (4DL14-5/38), the NDI Polaris optical tracking system and a phantom bone submerged in water that was previously scanned in a CT to obtain a high resolution ground truth model. We recorded 30 images of the femur covering the complete distal geometry apart from the articulating surfaces. This mimics clinical conditions, as the knee joint cavity of a healthy human being is not visible due to acoustic shadowing.

We conduct two in-vitro experiments: The first is the baseline experiment. For the second experiment, the individual images are registered to the ground truth mesh in order to eliminate all tracking errors. This allows determining the influence of the tracking errors on the reconstruction results. We use a common set of algorithmic parameters for all experiments.

The in-vivo experiment utilizes the same hardware, however due to motion artifacts the images were manually registered to the ground truth obtained from an MRI of the patient. Only 11 images, not depicting the distal as well as dorsal areas are recorded, creating very challenging conditions.

As both, the phantom bone as well as the patient's bone, are very similar to the statistical mean shape, we repeat all experiments but initialize the first three mode weights of the statistical shape model with deviating values of two standard deviations. This simulates a situation where the patient has a knee geometry that differs strongly from the average knee. All shapes can be found in Figure 1.

For evaluation, the shape returned by the algorithm is cropped to the areas relevant for implant planning: The condyles as well as a narrow outline all around the bone where cuts would be made. Subsequently this cropped shape is registered to the ground truth with the iterative closest point algorithm (ICP). This minimizes any alignment error and isolates the shape mismatch error. Finally, the average as well as the maximal surface distance error (SDE) is computed.

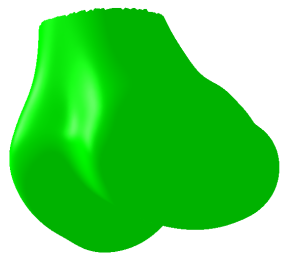

(a)

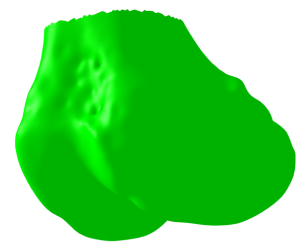

(b)

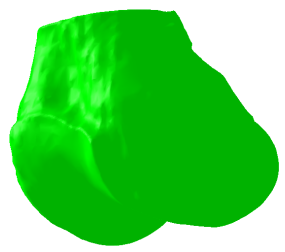

(c)

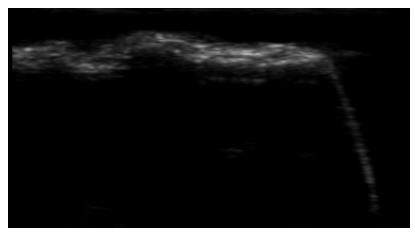

(g) 


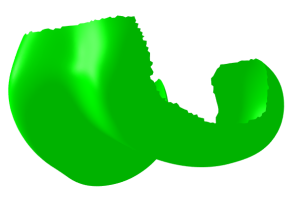

(d)

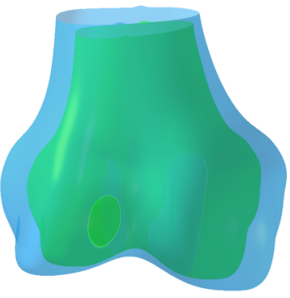

(e)

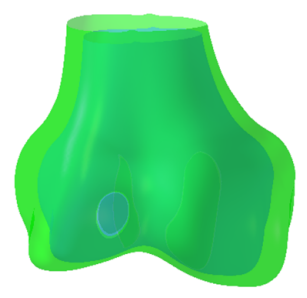

(f)

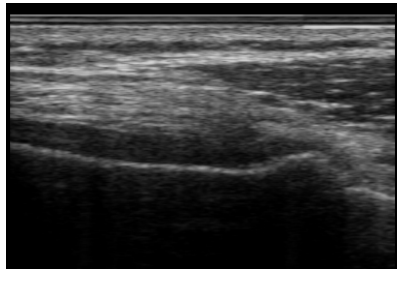

(h)

Figure 1 The initial mean shape (a), the ground truth mesh of the in-vitro (b) and in-vivo (c) experiment, the evaluation mask (d) as well as the mean shape (blue) overlaid with its shrank (e) and enlarged (f) variation (green). Note the similarity of the mean shape and the ground truths in size and shape. Sub-figure (g) and (h) show example image slices of the in-vitro and in-vivo US volumes respectively. See colored figure online.

\section{Results}

Table 1 shows the surface distance error of the different setups and initializations as well as the error before execution of the IPASM search. For the in-vitro experiments, the baseline results in no notable improvement of the reconstruction. Even without any tracking error present ('Registered'), the algorithm achieves only a small refinement of the bone surface. However, given a deviating initial shape the average SDE can be reduced by a large amount. The in-vivo evaluation confirms these findings however performs noticeably worse due to wrong soft-tissue correspondences.

\begin{tabular}{llllllll}
\hline \multirow{2}{*}{ SDE $(\mathrm{mm})$} & \multicolumn{2}{c}{ Original shape } & \multicolumn{2}{c}{ Shrank shape } & \multicolumn{2}{c}{ Enlarged Shape } \\
& & Avg. & Max. & Avg. & Max. & Avg. & Max. \\
\hline In-vitro & Initial Mean Shape & 0.68 & 2.9 & 2.07 & 9.63 & 3.7 & 10.84 \\
& Baseline & 0.72 & 3.72 & 1.35 & 4.27 & 1.26 & 4.26 \\
& Registered & 0.51 & 3.13 & 0.6 & 6.68 & 0.57 & 2.1 \\
\hline \multirow{2}{*}{ In-vivo } & Initial Mean Shape & 0.72 & 3.57 & 2.38 & 10.7 & 2.68 & 8.75 \\
& Baseline & 0.87 & 4.29 & 1.58 & 7.39 & 1.92 & 8.24 \\
\hline
\end{tabular}

Table 1 Results of the bone reconstruction. Three in-vitro setups are evaluated - the SDE of the mean shape before ('Initial Mean Shape') and after ('Baseline') execution of the IPASM, as well as another record that was registered to the ground truth via ICP to simulate a perfect tracking system ('Registered'). For the in-vivo experiment, the SDE before and after execution of the IPASM are presented.

\section{Discussion}

Looking at the in-vitro experiments in the first column of Table 1 ('Original Shape') the IPASM search is not able to reduce the SDE compared to the unaltered statistical shape model. This is due to the phantom body being very similar to the mean shape. Given a perfect registration of the images onto the ground truth, small improvements are possible. The subsequent experiments with strongly altered shapes however demonstrate the potential of the IPASM search: The baseline method achieves a SDE of roughly $1.3 \mathrm{~mm}$ on average and reduces the maximal error strongly to $4.3 \mathrm{~mm}$, which is comparable to the results by [Tadross 2012]. In contrast to our work however, they registered the recorded images prior to shape extraction, resulting in a setup similar to the 'Registered' case. Here the presented method can lower the average error to submillimeter range. While this performance still falls short to segmentation results presented in [Hacihaliloglu et al. 2008], the IPASM is able to not only segment but also register and extrapolate at the same time. For the in-vivo evaluation, the average SDE can be reduced although the performance drops noticeably. 
Overall, the maximal error is too high for planning processes in the medical context. Even given a perfect registration in an in-vitro setup, it cannot be reduced to an acceptable amount of below $3 \mathrm{~mm}$ for implant planning. Construction patient-specific instruments even requires sub-millimeter precision. The obvious weakness of the algorithm is the very simple segmentation strategy based on pixel intensity. However other segmentation methods like Shadow Peak or Phase Symmetry [Pandey et al. 2018] can be easily incorporated. Future work should therefore focus on preprocessing.

\section{References}

[Anas et al. 2016] Anas, E. M. A; Seitel, A; Rasoulian, A; John, P. S; Ungi, T; Lasso, A; DARRAS, K; Wilson, D; LeSSOWAY, V. A; Fichtinger, G.: Registration of a statistical model to intraoperative ultrasound for scaphoid screw fixation. In: International journal of computer assisted radiology and surgery, 11 (2016), 6: 957-965

[Golan et al. 2016] Golan, D; Donner, Y; MANSI, C; JAREMKo, J; RAMachandran, M.: Fully Automating Graf's Method for DDH Diagnosis Using Deep Convolutional Neural Networks. In: Deep Learning and Data Labeling for Medical Applications: Springer (2016): 130-141

[Hacihaliloglu 2017] HACIHALILOGLU, I.: Ultrasound imaging and segmentation of bone surfaces. A review. In: Technology, 5 (2017), 02: 74-80

[Hacihaliloglu et al. 2008] Hacihaliloglu, I; Abugharbien, R; Hodgson, A; Rohling, R.: Bone segmentation and fracture detection in ultrasound using 3D local phase features. In: International Conference on Medical Image Computing and Computer-Assisted Intervention (2008): 287-295

[Hänisch et al. 2017] HÄNISCH, C; HOHLMANN, B; RADERMACHER, K.: The interleaved partial active shape model search (IPASM) algorithm - Preliminary results of a novel approach towards 3D ultrasound-based bone surface reconstruction. In: Radermacher, K. \& Rodriguez Y Baena, F. (eds.): CAOS2017. EPiC Series in Health Sciences. Aachen, Germany (2017) (EPiC Series in Health Sciences): 399-406

[Litjens et al. 2017] Litjens, G; Kooi, T; Bejnordi, B. E; Setio, A. A. A; Ciompi, F; Ghafoorian, M; VAN DER LAAK, J. A; VAN GINNEKEN, B; SÁNCHEZ, C. I.: A survey on deep learning in medical image analysis. In: Medical image analysis, 42 (2017): 60-88

[Masoumi et al. 2017] MASOUMI, N; XIAO, Y; RIVAZ, H. (eds.): MARCEL (Inter-Modality Affine Registration with CorrELation Ratio). An Application for Brain Shift Correction in UltrasoundGuided Brain Tumor Resection: Springer (2017)

[OECD 2017] OECD, -: Hip and knee replacement. In: DEVELOPMENT, O. F. E. C.-O. A. (ed.): HEALTH AT A GLANCE 2017. Oecd indicators. Paris: ORGANIZATION FOR ECONOMIC (2017) (Health at a Glance): 178-179

[Pandey et al. 2018] Pandey, P; Guy, P; Hodgson, A. J; Abugharbieh, R.: Fast and automatic bone segmentation and registration of 3D ultrasound to CT for the full pelvic anatomy: a comparative study. In: International journal of computer assisted radiology and surgery, 13 (2018), 10: 1515-1524

[Tadross 2012] TADross, R. A. M.: A Novel Imaging System for Automatic Real-Time 3D PatientSpecific Knee Model Reconstruction Using Ultrasound RF Data (2012)

[Villa et al. 2018] Villa, M; Dardenne, G; NASAn, M; Letissier, H; Hamitouche, C; Stindel, E.: FCN-based approach for the automatic segmentation of bone surfaces in ultrasound images. In: International journal of computer assisted radiology and surgery, 13 (2018), 11: 1707-1716

[Wang et al. 2018] Wang, P; PAtel, V. M; Hacihaliloglu, I. (eds.): Simultaneous Segmentation and Classification of Bone Surfaces from Ultrasound Using a Multi-feature Guided CNN: Springer (2018) 